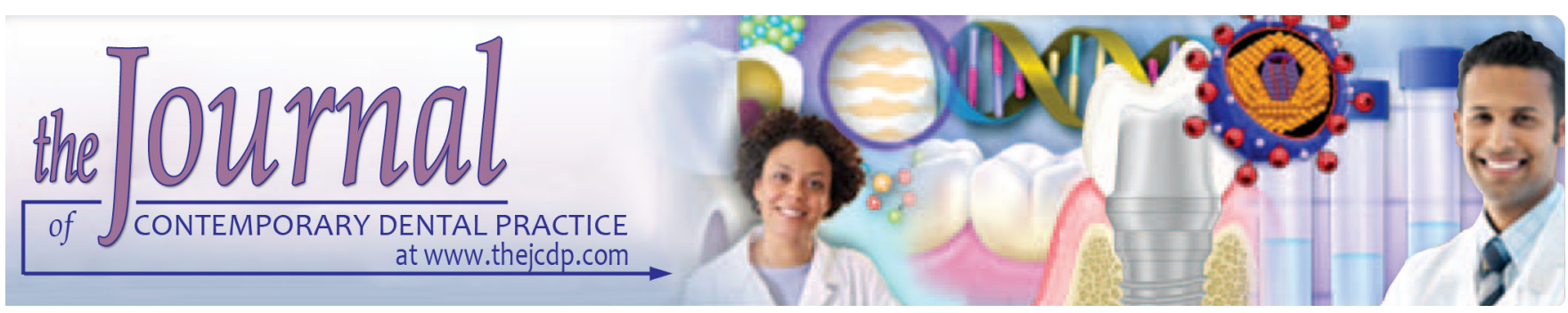

\title{
Assessment of Sirtuin 3 and Sirtuin 4 Level in Patients with Diabetes Mellitus and Periodontitis: A Clinical Study
}

\author{
${ }^{1}$ Rashmi Laddha, ${ }^{2}$ Monica Mahajania, ${ }^{3}$ Amruta Khadse, ${ }^{4}$ Rajat Bajaj, ${ }^{5}$ Rashmi Jawade, ${ }^{6}$ Shashwati Choube
}

\begin{abstract}
Aim: Periodontitis is considered as infection in periodontal supporting structure leading to tooth mobility and ulcerated periodontal pockets. The present study was conducted to assess Sirtuin 3 (SIRT 3) and SIRT 4 level in patients with diabetes mellitus (DM) and periodontitis.

Materials and methods: The present study was conducted on 60 subjects. Subjects were divided into four groups, groups I to IV. Each group comprised of 15 subjects. In all subjects, fasting blood glucose level was assessed. Plaque index (PI), bleeding on probing (BOP), gingival index (GI), and clinical attachment level (CAL) were measured. The SIRT 3 and SIRT 4 were estimated by Western blot analysis.
\end{abstract}

Results: In group I, mean age was $44.13 \pm 1.35$ years, in group II, it was $43.53 \pm 1.45$ years, in group III it was $43.93 \pm 1.22$ years, and in group IV, it was $44.47 \pm 0.74$ years. The mean BOP score was significantly higher in group IV $(5.74 \pm 0.30)$ compared with group I (1.92 \pm 0.44$)$, group II $(2.25 \pm 0.22)$, and group III $(5.31 \pm 0.54)$. A statistically significant $(p<0.001)$ difference was observed in mean PI score in group I ( $2.25 \pm 0.23)$, group II (2.26 \pm 0.13$)$, group III $(4.37 \pm 0.60)$, and group IV $(3.25 \pm 0.16)$. Mean GI score was significantly higher in group IV $(8.89 \pm 0.89)$ as compared with group I $(0.78 \pm 0.23)$, group II $(0.95 \pm 0.18)$, and group III (8.69 \pm 1.13$)$. A statistically significant difference was seen in mean CAL in group III $(5.66 \pm 0.64)$ and group IV

\footnotetext{
${ }^{1,5}$ Department of Periodontics, Dr Rajesh Ramdasji Kambe Dental College and Hospital, Akola, Maharashtra, India

${ }^{2}$ Department of Periodontics, Dr Hedgewar Smruti Rugna Seva Mandal Dental College and Hospital, Parola, Maharashtra, India

${ }^{3}$ Department of Endodontics, Vidya Shikshan Prasarak Mandals Dental College and Hospital, Nagpur, Maharashtra, India

${ }^{4}$ Private Practitioner, Consultant Orthodontist

${ }^{6}$ Department of Oral Medicine and Radiology, VYWS Dental College \& Hospital, Amravati, Maharashtra, India

Corresponding Author: Rashmi Laddha, Department of Periodontics, Dr Rajesh Ramdasji Kambe Dental College and Hospital, Akola, Maharashtra, India, Phone: +919501544977 e-mail: drrashmirdaga@gmail.com
}

(6.37 \pm 0.30$)$. Mean fasting blood sugar $(\mathrm{mg} / \mathrm{dL})$ in group I was $80.40 \pm 13.05$, in group II, it was $160.40 \pm 27.20$, in group III, it was $77.00 \pm 12.78$, and in group IV, it was $264.20 \pm 53.17$. The nonsignificant mean expression of SIRT 3 was seen in group I $(29.20 \pm 3.14)$, group II $(29.19 \pm 2.18)$, group III $(28.89 \pm 2.77)$, and group IV $(29.59 \pm 5.82)$. In group I, the mean level of SIRT 4 was $28.93 \pm 12.55$, in group II, it was $28.82 \pm 9.14$, in group III, it was $28.88 \pm 6.03$, and in group IV, it was $29.05 \pm 10.68$.

Conclusion: Association of DM and periodontitis is well known. The SIRT 3 and SIRT 4 are useful indicators of glycemic level in patients with DM.

Clinical significance: The SIRT 3 and SIRT 4 in DM show variation in their level. Early assessment may be proved beneficial in patients who are not responding to other drugs.

Keywords: Diabetes mellitus, Periodontitis, Sirtuin.

How to cite this article: Laddha R, Mahajania M, Khadse A, Bajaj R, Jawade R, Choube S. Assessment of Sirtuin 3 and Sirtuin 4 Level in Patients with Diabetes Mellitus and Periodontitis: A Clinical Study. J Contemp Dent Pract 2018;19(10):1199-1203.

Source of support: Nil

Conflict of interest: None

\section{INTRODUCTION}

Periodontitis is considered by infection in periodontal supporting structure leading to tooth mobility resulting in bacteremia. This is due to the presence of epithelial cells that show ulcerated periodontal pockets. It causes both local and systemic inflammatory host-immune response. Numerous studies have been conducted which correlate periodontitis and systemic diseases. ${ }^{1}$ It is stated that periodontal diseases are the leading cause of initiation and progression of systemic diseases. It has been a matter of interest to establish the relation between periodontitis and systemic health. As a local oral inflammatory disease, periodontitis may induce and promote systemic inflammation that initiates cardiovascular disease, pulmonary disease, rheumatoid arthritis, and DM. ${ }^{2}$ 
Among systemic diseases, DM is a common one which has a link with periodontitis. It is a metabolic disorder characterized by hyperglycemia due to defective secretion or activity of insulin. It is divided into insulindependent DM/type I DM and noninsulin-dependent $\mathrm{DM} /$ type II DM. Diabetes mellitus is analyzed by measuring glycated hemoglobin levels in blood, whereas sequential fasting plasma glucose levels is $7 \mathrm{mmol} / \mathrm{L}$ or more. ${ }^{3}$

The regulation of osteogenic differentiation in human periodontal ligament cells plays an imperative role for the development of new strategies for treating periodontitis. The exact mechanism by which diabetes affects periodontal tissues is not fully explained. It has been observed that there is altered immune-inflammatory response to bacterial pathogens leading to the disease. The SIRT 3 is a mitochondrial localized sirtuin which helps in glucose metabolism by increasing insulin sensitivity. Nasrin et $\mathrm{al}^{4}$ observed that SIRT 3 stimulates the ketogenesis by activating acetyl-coenzyme A synthetase in the mammalian cells. Thus, we can hope that SIRT 3 might play an important role for the enhanced ketogenesis seen during diabetes.

The SIRT 4 that takes part in glucose metabolism has an additional adenosine diphosphate (ADP)-ribosyl transferase activity which is concerned with telomere maintenance, genomic stability, and longevity. It ADPribosylates and inhibits the mitochondrial glutamate dehydrogenase (GDH). The GDH controls amino acidstimulated insulin secretion by regulating glutamine and glutamate oxidative metabolism. It is found that SIRT 1 and SIRT 4 appear to work in opposite directions to control insulin secretion as both the localization and the function of SIRT 4 are different. ${ }^{5}$

Although there is lack of sufficient information regarding the role of sirtuins in the osteogenic potential of human periodontal ligament cells, it is hypothesized that sirtuins regulate differentiation of human periodontal ligament cells and may play a role in periodontal bone regeneration.

So, the present study was conducted to assess the SIRT 3 and SIRT 4 level in patients with DM and periodontitis and its therapeutic efficiency in periodontitis.

\section{MATERIALS AND METHODS}

The present study was conducted on 60 subjects. All were informed regarding the study and written consent was obtained. Ethical clearance was taken prior to the study from the institutional ethical committee. Subjects with history of smoking and those on calcium channel blockers were excluded.
General information, such as name, age and gender was recorded. Subjects were divided into four groups, I, II, III, and IV, comprising 15 subjects each.

Group I: Healthy control subjects (nondiabetic; nonperiodontitis)

Group II: Diabetes without periodontitis

Group III: Periodontitis without diabetes

Group IV: Periodontitis with diabetes

In all subjects, fasting blood glucose level was assessed. Blood samples were obtained from all subjects and processed for assessment of glycated hemoglobin ( $\mathrm{HbA1C}$ ) level. If it was $<7$, they were considered to be controlled diabetics and those with $\mathrm{HbA} 1 \mathrm{C}$ level $>7$ were poorly controlled diabetics. Subjects were subjected to PI, BOP index, GI, and CAL.

Plaque index was measured at mesial, lingual, distal, and buccal site and scores given were $0,1,2$, and 3 . The $\mathrm{BOP}$ was measured at buccal and mesiobuccal sites. If the percentage of sites with BOP was less than 30 of all probed sites, it was defined as local bleeding. If the percentage of sites with BOP was higher than 30 of all probed sites, then it was considered as general BOP. Gingival index (Loe and Silness) was evaluated by periodontal probe. The GI score was marked as mild (0.1 to 1.0 ), moderate (1.1 to 2.0$)$, or severe (2.1 to 3.0). Clinical attachment level was calculated by adding gingival recession (GR) and probing depth.

In all subjects, $2 \mathrm{~mL}$ of blood was taken in a vial. For the estimation of SIRT 3 and SIRT 4, Western blot analysis was performed. Results thus obtained were subjected to statistical analysis using chi-square test and post hoc analysis using Tukey's test. A p-value less than 0.05 was considered significant.

\section{RESULTS}

The mean \pm standard deviation (SD) age of group I was $44.13 \pm 1.35$ years, that of group II $43.53 \pm 1.45$ years, for group III, it was $43.93 \pm 1.22$ years, and for group IV, it was $44.47 \pm 0.74$ years. The difference was statistically nonsignificant ( $p$ 0.219) (Graph 1). Out of 15 subjects in each group, males were 10 and females were 5. Graph 2 shows that mean BOP score in group I was $1.92 \pm 0.44$, in group II, it was $2.25 \pm 0.22$, in group III, it was $5.31 \pm 0.54$, and $5.74 \pm 0.30$ in group IV. The difference was statistically significant (p 0.001) (Graph 2). Mean PI score in group I was $2.25 \pm 0.23$, in group II, it was $2.26 \pm 0.13$, in group $\mathrm{III}$, it was $4.37 \pm 0.60$, and in group IV, it was $3.25 \pm 0.16$. The difference was statistically significant $(p<0.001)$. The post hoc analysis using Tukey's test revealed that the difference between groups I and II and between groups III and IV was statistically nonsignificant $(\mathrm{p}>0.05)$. However, the difference of mean PI scores between groups I and II differed significantly from that of groups III and IV. The 


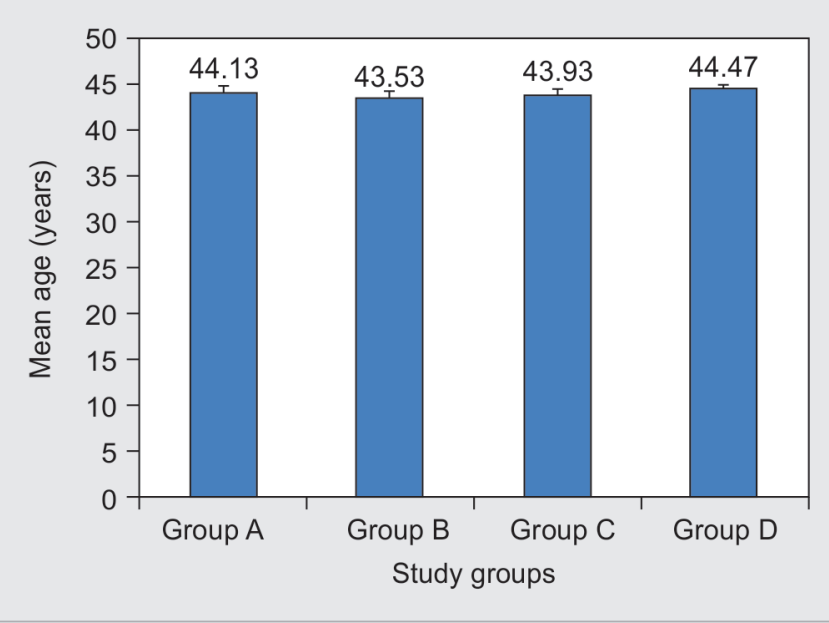

Graph 1: Mean age of patients in study groups

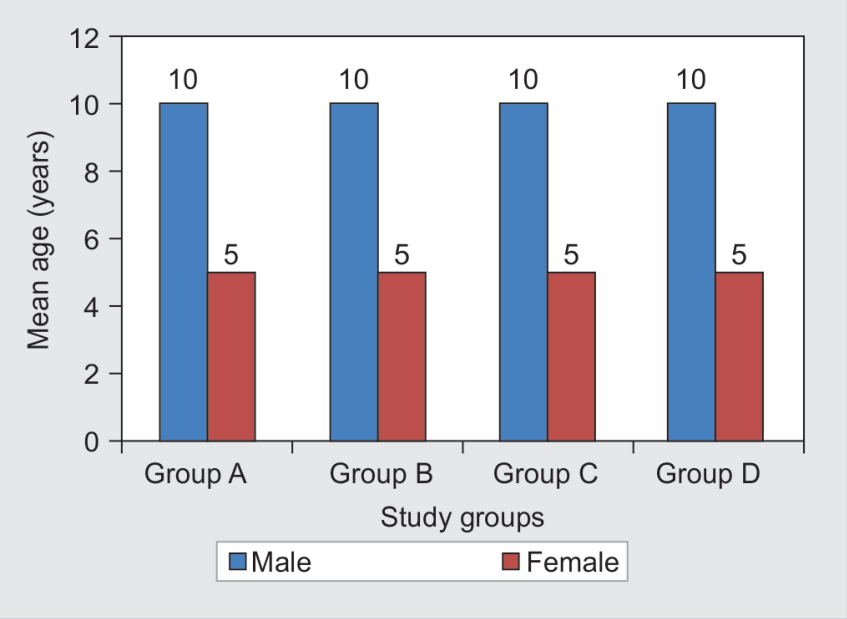

Graph 2: Gender-wise distributions of patients

Table 1: Comparison of BOP, $\mathrm{PI}$, and $\mathrm{GI}$ score in study groups

\begin{tabular}{llllll}
\hline Parameters & Group I $(n=15)$ & Group II $(n=15)$ & Group III $(n=15)$ & Group IV $(n=15)$ & $p$-value \\
\hline BOP & $1.92 \pm 0.44$ & $2.25 \pm 0.22$ & $5.31 \pm 0.54$ & $5.74 \pm 0.30$ & 0.001 \\
PI & $2.25 \pm 0.23$ & $2.26 \pm 0.13$ & $4.37 \pm 0.60$ & $3.25 \pm 0.16$ & 0.001 \\
GI & $0.78 \pm 0.23$ & $0.95 \pm 0.18$ & $8.69 \pm 1.13$ & $8.89 \pm 0.89$ & 0.001 \\
\hline
\end{tabular}

mean GI score in group I was $0.78 \pm 0.23$, in group II, it was $0.95 \pm 0.18$, in group III, it was $8.69 \pm 1.13$, and $8.89 \pm 0.89$ in group IV. The difference was statistically significant $(\mathrm{p}<0.001)$. The post hoc analysis using Tukey's test revealed that the difference between groups I and II and between groups III and IV was statistically nonsignificant $(p>0.05)$. Also the difference was statistically insignificant. However, the difference of mean GI scores between groups I and II differed significantly from that of groups III and IV (Table 1).

The descriptive statistics for CAL for two study groups: The mean CAL in group III was $5.66 \pm 0.64$, while in group IV, it was $6.37 \pm 0.30$. The difference was statistically significant (p 0.001) (Table 2).

The descriptive statistics for fasting blood sugar across four study groups: The mean fasting blood sugar

Table 2: Comparison of CAL between two study groups

\begin{tabular}{llll}
\hline$C A L$ & Group III $(n=15)$ & Group IV $(n=15)$ & $p$-value \\
\hline Mean \pm SD & $5.66 \pm 0.64$ & $6.37 \pm 0.30$ & 0.001 \\
\hline
\end{tabular}

$(\mathrm{mg} / \mathrm{dL})$ in group I was $80.40 \pm 13.05$, in group II, it was $160.40 \pm 27.20$, in group III, it was $77.00 \pm 12.78$, and in group IV, it was $264.20 \pm 53.17$. The difference was statistically significant (p 0.001) (Table 3).

The mean expression of SIRT 3 in group I was $29.20 \pm 3.14$, while in group II, it was $29.19 \pm 2.18$, group III, it was $28.89 \pm 2.77$, and group IV, it was $29.59 \pm 5.82$. The difference was statistically nonsignificant ( $p$ 0.972). The mean expression of SIRT 4 in group I was $28.93 \pm 12.55$, in group II, it was $28.82 \pm 9.14$, in group III, it was $28.88 \pm 6.03$, and in group IV, it was $29.05 \pm 10.68$ (Table 4).

\section{DISCUSSION}

Periodontitis is a disease of periodontium in which there is considerable tooth mobility, pocket formation, and GR. This is due to plaque accumulation in subgingival and supragingival tooth area. Plaque contains considerable bacteria leading to localized infection. The pathogenesis of periodontal disease has been demonstrated in different

Table 3: Comparison of fasting blood glucose in study groups

\begin{tabular}{llllll}
\hline Fasting blood sugar $(\mathrm{mg} / \mathrm{dL})$ & Group I $(n=15)$ & Group II $(n=15)$ & Group III $(n=15)$ & Group IV $(n=15)$ & $p$-value \\
\hline Mean \pm SD & $80.40 \pm 13.05$ & $160.40 \pm 27.20$ & $77.00 \pm 12.78$ & $264.20 \pm 53.17$ & 0.001 \\
\hline
\end{tabular}

Table 4: Comparison of mean SIRT 3 and SIRT 4 levels in study groups

\begin{tabular}{llllll}
\hline Sirtuins & Group I & Group II & Group III & Group IV & $p$-value \\
\hline SIRT 3 & $29.20 \pm 3.14$ & $29.19 \pm 2.18$ & $28.89 \pm 2.77$ & $29.59 \pm 5.82$ & 0.972 \\
SIRT 4 & $28.93 \pm 12.55$ & $28.82 \pm 9.14$ & $28.88 \pm 6.03$ & $29.05 \pm 10.68$ & 0.999 \\
\hline
\end{tabular}


methods, but little is known about the effect of periodontal disease on insulin resistance and glucose metabolism. Most of the studies have demonstrated that DM is a potential factor for periodontal inflammatory changes. Periodontal diseases, such as gingivitis and periodontitis are identified to be linked with remarkable rise in inflammatory cytokines and chemokines. ${ }^{6}$

Chances to periodontitis are increased when there is an enhanced level of interleukins, such as IL1 and IL1 gene polymorphism. Epigenetics and environmental factors changed the expression of deoxyribonucleic acid transcription and through these factors, an individual's predisposition for inflammatory response is determined. In a longitudinal study in patients with type II diabetes, the effect of periodontitis on deaths from cardiovascular complications and diabetic nephropathy has been investigated. Age- and sex-adjusted death rates were 28.4 times higher in subjects with severe periodontitis, 3.7 times higher in subjects with moderate periodontitis, and only 19.6 times in subjects with no/mild periodontitis. ${ }^{7}$

In the present study, we included 60 subjects which were divided into four groups of 15 each. Each group comprised of 10 males and 5 females. We found that mean \pm SD age of group I was $44.13 \pm 1.35$ years, for group II, it was $43.53 \pm 1.45$ years, group III, it was $43.93 \pm 1.22$ years, and group IV, it was $44.47 \pm 0.74$ years.

Saito et al. ${ }^{8}$ conducted a study and divided subjects into three groups. Group I comprised of subjects with normal glucose tolerance, group II had subjects with impaired glucose tolerance, and group III had subjects with no glucose tolerance (diabetics). The author observed an increase in mean pocket depth in group III (diabetics). It has been observed that hyperglycemia causes increase in oxidative stress which leads to imbalance between reactive oxygen species and antioxidants. This imbalance promotes accumulation of advanced glycation end products (AGE). The binding of AGE to their receptors promotes intracellular events that intensify the production of proinflammatory cytokines, chemokines, and cell adhesion molecules. ${ }^{9}$

It has been observed that diabetes further promotes periodontitis through an exaggerated inflammatory response to the periodontal microflora. The increased destruction of periodontal tissue seen in diabetic patients is due to enhanced inflammation in the disease causing destruction and decreased repair of damaged tissues. Periodontal disease has been associated with higher levels of inflammatory mediators, such as tumor necrosis factor- $\alpha$, IL-1 $\beta$ in people with diabetes. ${ }^{10}$

In the present study, we assessed the mean fasting blood sugar (mg/dL) in group I, which was $80.40 \pm 13.05$, in group II, it was $160.40 \pm 27.20$, in group III, it was $77.00 \pm 12.78$, and in group IV, it was $264.20 \pm 53.17$. The difference was found to be significant. This is in agreement with Ebersole and Cappelli. ${ }^{11}$ In the current study, BOP in all groups was assessed. It has been found that mean BOP score in group I was $1.92 \pm 0.44$, in group II, it was $2.25 \pm 0.22$, in group III, it was $5.31 \pm 0.54$, and $5.74 \pm 0.30$ in group IV. This is in agreement with Sima and Glogauer. ${ }^{12}$

Plaque index was evaluated in all the subjects which showed the mean PI score of $2.25 \pm 0.23,2.26 \pm 0.13$, $4.37 \pm 0.60$, and $3.25 \pm 0.16$ in groups I, II, III, and IV respectively. We found that mean GI in group I was $0.78 \pm 0.23$, in group II, it was $0.95 \pm 0.18$, in group III, it was $8.69 \pm 1.13$, and $8.89 \pm 0.89$ in group IV. This is in agreement with Lamster et al. ${ }^{13}$ study which showed that the mean CAL in group III was $5.66 \pm 0.64$, while in group IV, it was $6.37 \pm 0.30$. Histone deacetylases, such as SIRT 3 and SIRT 4 are enzymes that play an important role in DM. These are nicotinamide-adenine dinucleotide-dependent deacetylases. The SIRT 3 helps in increasing insulin sensitivity and decreasing blood glucose level. The SIRT 4 has ADP ribosylase activity, which inactivates GDH and in turn inhibits insulin secretion in pancreatic b-cells. ${ }^{14}$

We found that the mean expression of SIRT 3 in group I was $29.20 \pm 3.14$, in group II, it was $29.19 \pm 2.18$, in group III, it was $28.89 \pm 2.77$, and in group IV, it was $29.59 \pm 5.82$. Similarly, the mean expression of SIRT 4 in group I was $28.93 \pm 12.55$, in group II, it was $28.82 \pm 9.14$, in group III, it was $28.88 \pm 6.03$, and in group IV, it was $29.05 \pm 10.68$. This is in agreement with Haigis and Sinclair. ${ }^{15}$

The low level of sirtuins may lead to DM. Recent studies have shown that therapy targeted at activating SIRT 3 has a beneficial effect in patients with DM. ${ }^{16}$ Sirtuin 1 has been shown to suppress bone breakdown through the direct activation of FOXO proteins through their deacetylation.

The sirtuin has been shown to activate superoxide dismutase 2 , resulting in the diminution of oxidative stress and the inhibition of inflammation-mediated bone loss. So, it can be presumed that it might play an important role as therapeutics for inflammation-induced periodontal bone loss. ${ }^{17}$

Lee et al. ${ }^{18}$ conducted a study to elucidate the expression of SIRT 1 and osteoblastic differentiation markers and suggested that SIRT 1 is a potent regulator of differentiation of human periodontal ligament cells and have an imperative clinical impact on regeneration of periodontal bone.

\section{CONCLUSION}

The association of DM and periodontitis is well known. The SIRT 3 and SIRT 4 are useful indicators of glycemic level in patients with DM, and sirtuins may play a therapeutic role in periodontitis. 


\section{CLINICAL SIGNIFICANCE}

Thus, the role of SIRT 3 and SIRT 4 in DM may be determined by the fact that the level of these may be increased or decreased depending upon the level of blood glucose in the body. This may prove beneficial in patients who are not responding to drugs. New research is required which can utilize the information regarding the role of sirtuins in DM and periodontitis.

\section{REFERENCES}

1. Mealey BL, Ocampo GL. Diabetes mellitus and periodontal disease. Periodontol 2000 2007;44:127-153.

2. Taylor JJ, Preshaw PM, Lalla E. A review of the evidence for pathogenic mechanisms that may link periodontitis and diabetes. J Clin Periodontol 2013 Apr;40 (Suppl 14):S113-S134.

3. Dandona P, Aljada A, Bandyopadhyay A. Inflammation: the link between insulin resistance, obesity and diabetes. Trends Immunol 2004 Jan;25(1):4-7.

4. Nasrin N, Wu X, Fortier E, Feng Y, Bare' OC, Chen S, Ren $\mathrm{X}, \mathrm{Wu} \mathrm{Z}$, Streeper RS, Bordone L. SIRT4 regulates fatty acid oxidation and mitochondrial gene expression in liver and muscle cells. J Biol Chem 2010 Oct 15;285(42):31995-32002.

5. Someya S, Yu W, Hallows WC, Xu J, Vann JM, Leeuwenburgh C, Tanokura M, Denu JM, Prolla TA. Sirt3 mediates reduction of oxidative damage and prevention of age-related hearing loss undercaloric restriction. Cell 2010 Nov 24;143(5):802-812.

6. Papapanou PN. Periodontal diseases: epidemiology. Ann Periodontol 1996 Nov;1(1):1-36.

7. Kornman KS, Giovinef FS. Genetic variations in cytokine expression: a risk factor for severity of adult periodontitis. Ann Periodontol 1998 Jul;3(1):327-338.

8. Saito T, Shimazaki Y, Kiyohara Y, Kato I, Kubo M, Iida M, Koga $T$. The severity of periodontal disease is associated with the development of glucose intolerance in non-diabetics: the Hisayama study. J Dent Res 2004 Jun;83(6):485-490.

9. Faria-Almeida R, Navarro A, Bascones A. Clinical and metabolic changes after conventional treatment of type 2 diabetic patients with chronic periodontitis. J Periodontol 2006 Apr;77(4):591-598.

10. Singh S, Kumar V, Kumar S, Subbappa A. The effect of periodontal therapy on the improvement of glycemic control in patients with type 2 diabetes mellitus: a randomized controlled clinical trial. Int J Diabetes Dev Ctries 2008 Apr;28(2): 38-44.

11. Ebersole JL, Cappelli D. Acute phase reactants in infections and inflammatory diseases. Periodontology 20002000 Jun;23:19-49.

12. Sima C, Glogauer M. Diabetes mellitus and periodontal diseases. Curr Diab Rep 2013 Jun;13(3):445-452.

13. Lamster IB, Lalla E, Borgnakke WS, Taylor GW. The relationship between oral health and diabetes mellitus. J Am Dent Assoc 2008 Oct;139 (Suppl):19S-24S.

14. Wilson AG. Epigenetic regulation of gene expression in the inflammatory response and relevance to common disease. J Periodontol 2008 Aug;79(8 Suppl):1514-1519.

15. Haigis MC, Sinclair DA. Mammalian sirtuins: biological insights and disease relevance. Annu Rev Pathol 2010;5: 253-295.

16. North BJ, Verdin E. Interphase nucleo-cytoplasmic shuttling and localization of SIRT2 during mitosis. PloS One 2007 Aug 29;2(8):e784

17. Chiu AV, Saigh MA, McCulloch CA, Glogauer M. The role of $\mathrm{NrF} 2$ in the regulation of periodontal health and disease. J Dent Res 2017 Aug;96(9):975-983.

18. Lee YM, Shin SI, Shin KS, Lee YR, Park BH, Kim EC. The role of sirtuin 1 in osteoblastic differentiation in human periodontal ligament cells. J Periodontal Res 2011 Dec;46(6):712-721. 